\title{
Adaptation and validation of the Nigerian Igbo Multidimensional Scale of Perceived Social Support in patients with chronic low back pain
}

Chinonso Nwamaka Igwesi-Chidobe ${ }^{1,2^{*}}$ (D), Sheila Kitchen ${ }^{2}$, Isaac Olubunmi Sorinola ${ }^{2}$ and Emma Louise Godfrey ${ }^{2,3}$

\begin{abstract}
Introduction: Social support may be important in the perpetuation of symptoms in chronic low back pain (CLBP). The multidimensional scale of perceived social support (MSPSS) is one of the best measures of social support with applicability in Africa.
\end{abstract}

Aims: The aims of this study were to translate, culturally adapt, test-retest, and assess cross-sectional psychometric properties of the Igbo-MSPSS.

Methods: Forward and backward translation of the MSPSS was done by clinicians and non-clinician translators and evaluated by a specialist review committee. The adapted measure was piloted amongst twelve adults with CLBP in rural Nigeria. Cronbach's alpha and McDonald's omega coefficient were used for investigating internal consistency. Intra-class correlation coefficient (ICC: two-way random effects model, average of raters' measurements, absolute definition of agreement) reflecting both the degree of correlation and agreement between measurements was used for the statistical investigation of test-retest reliability. Criterion validity of the adapted measure was investigated with the eleven-point box scale, back performance scale, Roland Morris Disability Questionnaire, and World Health Organisation Disability Assessment Schedule amongst 200 people with CLBP in rural Nigeria using Spearman's correlation analyses. Exploratory factor analyses conducted using Kaiser criterion and parallel analysis as methods for determining dimensionality were used to determine the structural validity of the adapted measure amongst the same sample of 200 rural dwellers.

Results: Igbo-MSPSS had excellent internal consistency (0.88) and ICC of 0.82. There were moderate correlations with measures associated with the social support construct. The same item-factor pattern in the three-dimensional structure (with Kaiser criterion) as in the original measure and a two-dimensional structure (with parallel analysis) were produced.

\footnotetext{
* Correspondence: chinonso.chidobe@unn.edu.ng

'Department of Medical Rehabilitation, Faculty of Health Sciences and

Technology, College of Medicine, University of Nigeria (Enugu Campus),

Enugu, Nigeria

2Department of Physiotherapy, School of Population Health Sciences, Faculty

of Life Sciences and Medicine, King's College London, London, UK

Full list of author information is available at the end of the article
}

(c) The Author(s). 2021 Open Access This article is licensed under a Creative Commons Attribution 4.0 International License, which permits use, sharing, adaptation, distribution and reproduction in any medium or format, as long as you give

appropriate credit to the original author(s) and the source, provide a link to the Creative Commons licence, and indicate if changes were made. The images or other third party material in this article are included in the article's Creative Commons licence, unless indicated otherwise in a credit line to the material. If material is not included in the article's Creative Commons licence and your intended use is not permitted by statutory regulation or exceeds the permitted use, you will need to obtain permission directly from the copyright holder. To view a copy of this licence, visit http://creativecommons.org/licenses/by/4.0/. 
Conclusions: Igbo-MSPSS is a measure of social support with some evidence of validity and reliability and can be used clinically or for research. Future studies are required to confirm its validity and reliability.

Keywords: Social support, Chronic pain, Chronic low back pain, Chronic disease, Cross-cultural, Rural, Nigeria, Africa

\section{Introduction}

Several definitions of social support abound. It could mean that someone is being cared for and loved (succour, nurture, affiliation); someone is esteemed and valued (recognition, respect); or that someone belongs to a network of supportive groups where communication and obligation are shared (group membership), or that someone perceives that these are available when needed (Pearson, 1986; Taylor, 2011). Evidence suggests that social support, such as in the form of supportive connection with others, a sense of belonging or being loved by/ important to others, and involvement in social groups, is beneficial to both mental and physical health either directly or indirectly via reduction of stress (Taylor, 2011; Thoits, 2011).

Few studies have examined the impact of social support on chronic low back pain (CLBP). A systematic review that investigated the influence of social support at work on the risk of occurrence of low back pain, recovery, and return to work found no effect of social support from co-workers, supervisors or general work on the risk of a new episode of low back pain. However, there were weak effects of support at work on pain recovery and return to work. Higher co-worker and general work support were associated with shorter recovery time and faster return to work (Campbell, Wynne-Jones, Muller, \& Dunn, 2013). A cross-sectional study found that the association between forward bending and low back pain intensity was modified by social support. Manual workers who reported low levels of social support and long duration of forward bending had higher levels of low back pain compared with similar workers who reported more social support and similar period of forward bending (Villumsen, Holtermann, Samani, \& Madeleine, 2016).

The multidimensional scale of perceived social support (MSPSS) is widely used; has good validity, reliability, and utility in terms of brevity and simplicity; includes social support from the significant other; and has applicability in Africa (Dambi et al., 2018; Ehrlich George, 2003; Zimet, Dahlem, Zimet, \& Farley, 1988; Zimet, Powell, Farley, Werkman, \& Berkoff, 1990). A systematic review involving 22 translations of the MSPSS into languages other than English, from 70 studies, found poor translation methods in 16 of the studies; and only 4 of the studies performed confirmatory factor analyses for confirmation of structural validity or longitudinal test-retest reliability (Dambi et al., 2018). There was good evidence for validity and reliability of the MSPSS from the few high-quality studies in that review. The original measure has high internal consistency and test-retest reliability (> 0.8), and correlations with quality-of-life measures (De Maria, Vellone, Durante, Biagioli, \& Matarese, 2018). Confirmatory factor analysis of the original measure in people with chronic diseases (De Maria et al., 2018) and a Spanish version amongst early retirees enrolled in university programmes (Lopez Ramos, Fernandez Munoz, Navarro-Pardo, \& Murphy, 2017) replicated the three-factor structure found in the original study (Zimet et al., 1988, 1990). Indeed, the most consistently reported structure of the MSPSS in cross-cultural adaptations is a three-factor solution which confirms the three distinct sources of support from family, friends, and significant others (Adamczyk, 2013; Dambi et al., 2018; Gabardo-Martins, Ferreira, \& Valentini, 2017; Laksmita, Chung, Liao, \& Chang, 2020; Trejos-Herrera, Bahamón, Alarcón-Vásquez, Vélez, \& Vinaccia, 2018) reported in the original scale (Zimet et al., 1988, 1990). In contrast, there have been fewer reports of two-factor (Chou, 2000; Lee, Moy, \& Hairi, 2017) and one-factor (Akhtar et al., 2010) structures of the MSPSS depending on cultural definitions of social support. A two-factor structure consisting of friend and family subscales have been extracted in cultures where family and significant others were combined into one, and regarded as one distinct source of social support, such as in Chinese and Malay cultures (Chou, 2000; Lee et al., 2017). In contrast, social support has been perceived as a unitary construct in Pakistani culture (Akhtar et al., 2010).

Qualitative research evidence suggests that social support may influence the impact of CLBP in rural Nigeria. For instance, the coping strategy 'escaping from others' appeared to be provoked by shame due to perceived negative perceptions from family and friends who were unsupportive of those living with CLBP in rural Nigeria (Igwesi-Chidobe, Kitchen, Sorinola, \& Godfrey, 2017; Igwesi-Chidobe, Sorinola, Kitchen, \& Godfrey, 2018). It is therefore pertinent to quantitatively investigate the influence of social support on CLBP disability in rural Nigeria using valid, reliable, and utilitarian instruments.

Therefore, the objectives of this study include

1. Translation and cultural adaptation of the MSPSS into the Nigerian Igbo

2. Investigation of the reliability of the Nigerian IgboMSPSS including test-retest reliability 
3. Examination of the criterion validity of the Nigerian Igbo-MSPSS

4. Inspection of the factor structure of the Nigerian Igbo-MSPSS

\section{Methods}

\section{Ethical concerns}

King's College London (Ref: BDM/13/14-99) and University of Nigeria Teaching Hospital (Ref: UNTH/CSA/ 329/Vol.5) gave ethical approval. The original developers of the questionnaire gave permission to adapt the measure.

\section{Study designs}

This study involved the translation and cultural adaptation of the Igbo-MSPSS, test and retest investigation of its reliability, and cross-sectional investigation of its validity.

\section{Outcome measures}

Multidimensional Scale of Perceived Social Support (MSPSS)

MSPSS is a patient-reported measure of subjectively assessed social support (Zimet et al., 1988). The scale has twelve items in total, with three subscales (family, friends, or significant other) of four items each, which can be added as a total score, or as subscales depending on the source of social support. There is a 7-point Likert scale in each item which has values between 1 (strongly disagree) to 7 (strongly agree). The minimum score for each subscale is 4 , and 28 is the maximum score. For a total scoring, 12 is the minimum score, and 84 is the maximum score. The higher the score, the higher the perceived social support. An internal consistency of 0.88 and test-retest reliability of 0.85 were found for the original scale (Zimet et al., 1988, 1990).

\section{Eleven-point box scale (BS-11)}

Pain was measured using the BS-11, which is a numeric scale for measuring pain intensity with a single item that has eleven numbers (from 0 to 10) enclosed in boxes (Hawker, Mian, Kendzerska, \& French, 2011; Jensen, Karoly, \& Braver, 1986). 'No pain' is a score of zero, whilst 10 is the highest intensity of pain possible. This measure is easy to comprehend and administer. It is probably the best outcome measure for pain in populations with limited literacy such as rural Nigeria (IgwesiChidobe, Kitchen, et al., 2017).

\section{Back performance scale (BPS)}

BPS is an objective (scored by an assessor) back painspecific measure of performance-based disability that assesses the disability domain of mobility (Strand, MoeNilssen, \& Ljunggren, 2002). Five physical performance tests that involve moving the trunk are performed. The tests are sock test, pick-up test, roll-up test, finger-tipto-floor test, and lift test. In the sock test, participants mimick putting on a sock normally from the sitting position. Participants attempt to pick up a piece of paper from the floor normally in the pick-up test. There is a slow roll-up from supine lying to a long sitting position with arms relaxed by the sides in the roll-up test. Whilst standing on the floor with feet $10 \mathrm{~cm}$ apart, participants bend forward as they keep their knees straight and attempt touching the floor with both fingertips. The space between the floor and the fingertips is subsequently computed in centimetres in the finger-tip-to-floor test. There is repetition of lifting a $5-\mathrm{kg}$ box from the floor to a $76-\mathrm{cm}$ table and back to the floor for $1 \mathrm{~min}$, and the number of lifts is subsequently documented in the lift test. Values range from 0 to 3 depending on the difficulty or ease with which each of the five tests are performed, with a total possible score of 15 (maximum disability) and lowest possible score of 0 (no disability). The measure has good psychometric properties with test-retest reliability of 0.91 , internal consistency of 0.73 , and moderate correlations with Roland Morris Disability Questionnaire (RMDQ) $(r=0.45)$ (Magnussen, Strand, \& Lygren, 2004; Myklebust, Magnussen, \& Inger Strand, 2007; Strand et al., 2002).

\section{Igbo Roland Morris Disability Questionnaire (Igbo-RMDQ)}

RMDQ is endorsed as a main outcome tool for back pain randomized controlled trials, systematic reviews and meta-analyses, and cost-effectiveness analyses. RMDQ is user friendly, understood easily by people with minimal education, and is the best measure for public health and population studies (Roland \& Fairbank, 2000). The original RMDQ (Roland \& Morris, 1983) has been cross-culturally adapted for Nigerian Igbo population-the Igbo-RMDQ (Igwesi-Chidobe, Obiekwe, Sorinola, \& Godfrey, 2019). It has twentyfour items with each having scores of either 0 or 1 . The higher the value, the greater the disability with 24 being the highest possible score and 0 indicating no disability. The cross-cultural adaptation confirmed content validity of the Igbo-RMDQ (Roland \& Fairbank, 2000). The Igbo-RMDQ has a Cronbach's alpha of 0.91; test-retest reliability of between 0.71 and 0.91 (Igwesi-Chidobe et al., 2019); moderate correlations with performance-based back painspecific disability and clinically important difference of 2-3-point change from baseline (Roland \& Fairbank, 2000). These support its internal consistency, test-retest reliability, construct validity, and responsiveness respectively. RMDQ aligns with the biopsychosocial disability model (World Health Organisation, 2001). 


\section{Igbo World Health Organisation Disability Assessment Schedule (Igbo-WHODAS 2.0)}

The 36-item interviewer-administered version of the WHODAS 2.0 is a comprehensive disability tool that measures disability within the ICF biopsychosocial model (World Health Organisation, 2001). It emphasizes all six areas of disability which include cognition, mobility, self-care, getting along with people, life activities, and participation including disability associated with work (Üstün, Kostanjsek, Chatterji, \& Rehm, 2010). The tool has been cross-culturally adapted for Nigerian Igbo population (Igwesi-Chidobe, Kitchen, Sorinola, \& Godfrey, 2020). As the measure is generic and comprehensive, it would enable comparisons across populations, conditions and an understanding of specific disability domains affected. The Igbo-WHODAS 2.0 has sound psychometric properties. The estimates for the Cronbach's alpha ranges between 0.80 and 0.90 ; test-retest reliability ranges between 0.80 and 0.90 ; and minimal detectable change ranges between 5.05 and 13.99 (IgwesiChidobe et al., 2020). The tool which has a simple and complex scoring method was used due to the low literacy levels in this population. For simple scoring, 'none' = 1 , 'mild' $=2$ 'moderate' $=3$, 'severe' $=4$, and 'extreme' $=$ 5 , and these are simply summed. However, the complex scoring method is preferable as it allows comparison across populations and conditions (Üstün et al., 2010) hence was utilized for this study. Complex scoring method weights each item, taking into consideration their levels of difficulty.

\section{Cross-cultural adaptation procedure}

Translation means paraphrasing the language of a questionnaire to another language. In contrast, cross-cultural adaptation comprises both translation and cultural adaptation to ensure that the same concept is reflected in the adapted instrument as in the original measure ensuring content validity of the adapted instrument.

\section{Participants for cross-cultural adaptation}

Translators were one clinical physiotherapist (native Igbo speaker, bilingual in English and Igbo) and three non-clinical translators including two Igbo linguistic experts (one native English speaker (bilingual in English and Igbo), one native Igbo speaker (bilingual in Igbo and English), and one English/Igbo linguistic expert). The clinical physiotherapist had 20 years of experience practising as a clinician in Nigeria. Expert committee members included one health psychologist and one academic physiotherapist from England practicing in the UK and one clinical psychologist and one clinical physiotherapist from the Igbo tribe practicing in Nigeria.

Piloting of the Igbo-MSPSS (qualitative assessment of content validity) was done with a convenience sample of adults living with CLBP in rural Nigeria. They received both verbal and written information about the study prior to obtaining informed consent, and all questionnaires were interviewer-administered by the researcher.

\section{Process for cross-cultural adaptation}

The original MSPSS was cross-culturally adapted adopting recommended evidence-based guidelines (Beaton, Bombardier, Guillemin, \& Ferraz, 2000; Mokkink et al., 2019; Wild et al., 2005) (Fig. 1).

Firstly, the original MSPSS was translated forwards from English to Igbo by a bilingual musculoskeletal physiotherapist and another bilingual professional translator from a non-clinical background. The forward translators were both native Igbo speakers but fluent in English. These Igbo translations resulted in FT1 and FT2 versions respectively. The physiotherapist had all the items explained to her to facilitate an understanding of the construct being assessed, whilst the items were not defined for the non-clinical translator to ensure that the language and expressions used in the translation reflected the routinely used language in the population.

Secondly, one Igbo FT-12 version was produced by the integration of $\mathrm{T} 1$ and $\mathrm{T} 2$ versions through a discussion between the two forward translators in a research meeting that was arbitrated by the bilingual lead author. Inconsistencies in the translations were recorded and compared with the original measure.

Thirdly, the Igbo FT-12 MSPSS version was back translated from Igbo to English by two non-clinical back translators, unfamiliar with the construct assessed by the original MSPSS. BT1 and BT2 MSPSS versions resulted from this back-translation process and validated the translation process, ensuring that the FT-12 MSPSS was reflecting the original meaning. One of the back translators was an English/Igbo linguistic expert proficient in the professional translation of tools and the other was a native English speaker, born in England to Nigerianborn Igbo parents.

Fourthly, the pre-final Igbo-MSPSS was produced by the expert review committee discussing the T1, T2, FT12, BT1, and BT2 MSPSS versions ensuring semantic, idiomatic, experiential, and conceptual equivalence with the original tool. The committee ensured that the words used in the Igbo translations reflected the original tool to confirm semantic equivalence. The committee devised alternative Igbo idioms and colloquialisms, where there was no equivalent Igbo expression of the original English idioms and colloquialisms. The committee ensured that items in the Igbo-MSPSS were experienced similarly in English and Igbo cultures and this guaranteed experiential equivalence. In order to ensure conceptual equivalence of the Igbo-MSPSS, the committee established that the words in the instructions, items, and response 


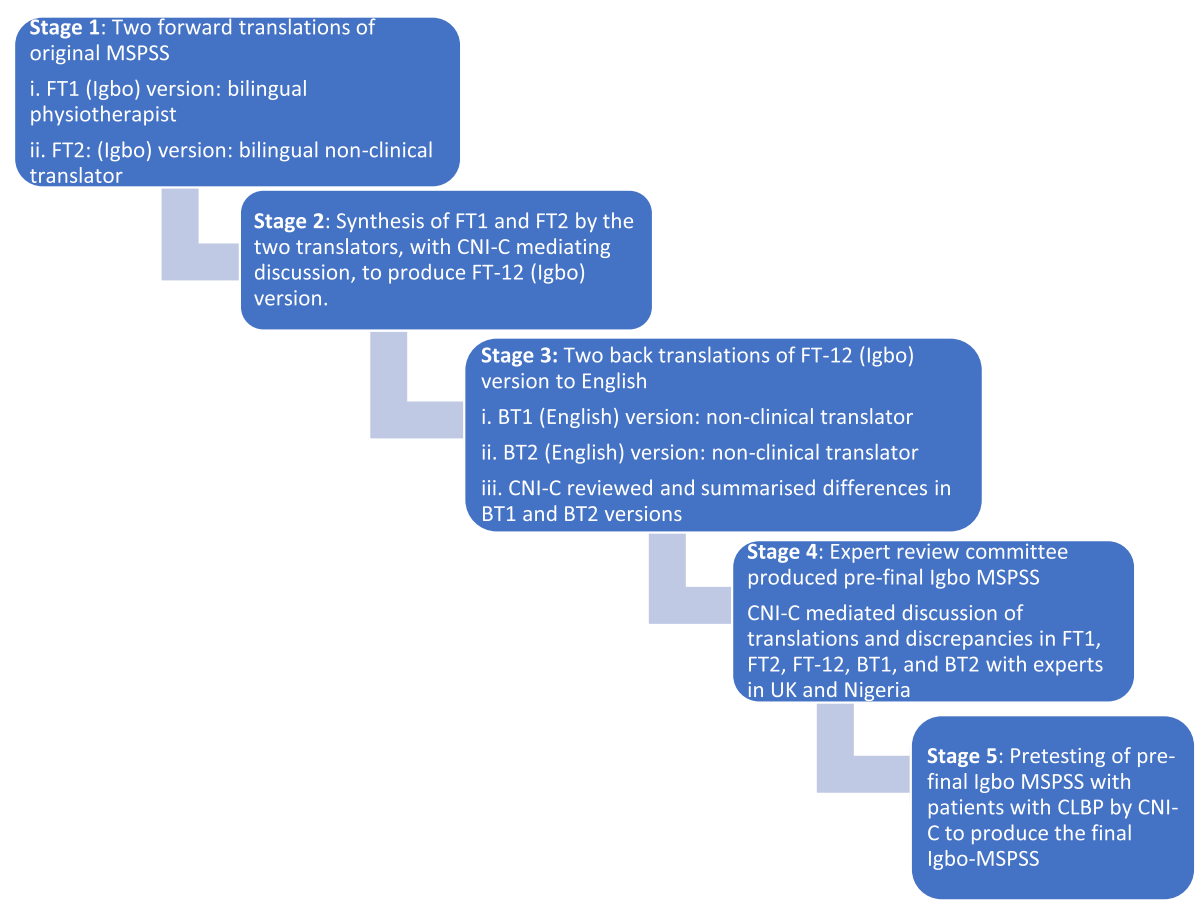

Fig. 1 Stages adopted in the cross-cultural adaptation of the original MSPSS into Nigerian Igbo. FT, forward translation; BT, back translation

choices had similar conceptual meanings in Igbo and English cultures. For instance, 'family' may signify the nuclear family in a western culture, whereas it may mean both the nuclear and extended family in rural Nigeria. The expert review committee determined that the Igbo wordings used in the final translation were unsophisticated enough to be understood by people with minimal education.

Finally, pre-final Igbo-MSPSS was piloted in rural Nigeria, among twelve adults living in rural Nigeria with CLBP. The lead author interviewer-administered the measures adopting a 'think-aloud' cognitive interviewing style. Participants actively verbalised their thoughts as they tried to answer each item following a reading out by the lead author. The lead author tried to understand if participants had difficulty understanding the IgboMSPSS, what they understood by each Igbo-MSPSS item, and the meaning of their chosen response and wrote down participants' responses as they were talking. This stage ensured that equivalence was maintained in the Nigerian context to produce the final Igbo-MSPSS, confirming content validity.

\section{Psychometric testing of Igbo-MSPSS Participants for test-retest reliability and criterion validity assessments}

For test-retest reliability investigation, ten community health workers (CHWs) were trained to collect data from 5 participants each. A convenience sample of 50 adults (18-69 years) with CLBP were recruited from rural and urban Enugu State communities for test-retest reliability assessment. Verbal and written information about the study was given to participants followed by informed consent before data collection. Sample size estimation was based on the recommendations for determining the sample size requirements for estimating the values of intra-class correlation coefficients (ICCs) (Bujang \& Baharum, 2017). A post hoc sample size estimation showed that this study was sufficiently powered. The least ICC value of 0.68 and the maximum ICC value of 0.90 found in this study require minimum sample sizes of 13 and 6 respectively, at $90 \%$ power and at 0.05 alpha level (Bujang \& Baharum, 2017).

Our study adopted an exploratory data-driven bottom-up approach in the inspection of the factor structure of the Igbo-MSPSS as well as its correlation coefficients. It aimed to explore if there would be any deviations in the structure of the Igbo-MSPSS and any correlations or lack thereof that would call into question its comparability with the original MSPSS. The sample size to achieve stable correlation estimates depends on the effect size, the width of the corridor of stability-that is the corridor around the true value where deviations are tolerated-and the requested confidence that the trajectory does not leave this corridor any longer (Schönbrodt \& Perugini, 2013, 2018). In typical scenarios, the sample size should approach 250 for stable correlation estimates, although it has been estimated that the point 
of stability is 161, after which the actual trajectory does not leave the corridor of stability (Schönbrodt \& Perugini, 2013, 2018). Regarding factor analysis, most studies have not recommended any absolute minimum sample size for factor analysis as this is dependent on several factors. Minimum sample sizes appear to be smaller for higher levels of communality and higher ratios of the number of variables to the number of factors (Mundfrom, Shaw, \& Ke, 2005). When the variables-to-factors ratio exceeds 6 , the minimum sample size begins to stabilize regardless of the number of factors or the level of communality (Mundfrom et al., 2005).

Investigation of validity involved a random representative sample of 200 participants, adults who were having CLBP in rural Enugu State communities (Igwesi-Chidobe, Coker, Onwasigwe, Sorinola, \& Godfrey, 2017).

Ten local government areas (LGAs) were randomly selected using multistage cluster sampling to represent rural Enugu State populations. Ten CHWs were trained for data collection from 20 participants living with CLBP in each LGA, giving a total of 200 participants. Participants with the 'red flags' for LBP which included underlying serious conditions including infection, cauda equina syndrome, spinal fracture, malignancy, spinal stenosis, and radiculopathy were excluded. Verbal and written information about the study were provided to participants after which informed consent was obtained.

\section{Psychometric testing procedure}

Training for data collection Interviewer administration was needed for data collection as a great proportion of rural Nigerians are illiterate. CHWs were identified from the University of Nigeria Teaching Hospital (UNTH) Enugu and trained for data collection. The manual used for training was based on the foundations of good survey design, directives by the questionnaire originators, review of the literature, and findings from the piloting of the questionnaire. The CHWs were trained for 2 weeks, for interviewer administration of questionnaires, and measurement of performance-based disability using the BPS. The aim of the training was to diminish measurement, non-response, sampling, and coverage errors. Using psychometrically sound instruments and avoiding asking participants leading questions or using gestures that could bias their responses reduced measurement error. Non-response errors were controlled through data collection from every recruited participant and ensuring that all questionnaire items were answered. Appropriate sampling technique and sample size reduced sampling error. Recruiting a representative sample of rural dwellers in the Enugu state reduced coverage error. CHWs were competent with the data collection protocol, before data collection in the field.

\section{Data collection}

An outcome measure booklet containing screening and demographic questions, and the Igbo self-report and objective measures was used by each $\mathrm{CHW}$ for data collection. Prior to being assessed and interviewed, participants were screened and asked to indicate on a body chart where they felt pain on their body. This process ensured that participants were having pain in the lower back. The CHWs then intervieweradministered the self-report measures. 'Flash cards' were used to present the Likert scales in each questionnaire item, and these were verbalised to participants. CHWs used the BPS to objectively measure performance-based disability.

Outcome tools were filled at baseline and completed a second time after 7 to 10 days following baseline assessment to assess test-retest reliability. The two measurements were completed by the same $\mathrm{CHW}$ for each participant.

Validity assessment involved completing the measures once using the cross-sectional design.

\section{Statistical analyses}

IBM SPSS version 22 and JASP version 0.14 .1 were used for data analyses. Normality of data were assessed visually and statistically.

\section{Reliability}

Reliability assesses the consistency with which an instrument measures a construct (Tavakol \& Dennick, 2011). Cronbach's alpha and McDonald's omega coefficient were used to assess internal consistency for the IgboMSPSS and its subscales/factors and rated as not acceptable $(<0.70)$, low $(0.70-0.80)$, moderate $(0.80-0.90)$, and high $(>0.90)$.

The intra-class correlation coefficient (ICC), commonly used to evaluate interrater, test-retest, and intrarater reliability is a desirable measure of reliability as it reflects both the degree of correlation and agreement between measurements (Koo \& Li, 2016). In contrast, historical methods of assessing reliability in the past such as Pearson correlation coefficient, paired t test, and Bland-Altman plot are limited in their applicability. Paired $t$ test and Bland-Altman plot are methods for analysing agreement, whilst Pearson correlation coefficient is only a measure of correlation. Therefore, these are nonideal measures of reliability (Koo \& $\mathrm{Li}, 2016$ ).

A two-way random effects model using the average of raters' measurements with an absolute definition of agreement between test-retest scores was used in the calculation of ICC. The recommended average of raters' measurements was the type of ICC utilised in this study since there were ten different assessors who collected data from the participants (Koo \& Li, 2016). A score of 
0.70 signified good test-retest reliability, 0.80 denoted very good test-retest reliability, and 0.90 means excellent test-retest reliability (Grotle, Brox, \& Vollestad, 2003; Shrout \& Fleiss, 1979).

Bland-Altman plots (Bland \& Altman, 1986) were used to investigate the visual agreement between test-retest measurements of the Igbo-MSPSS. This is achieved by graphically plotting mean Igbo-MSPSS score against the difference in the Igbo-MSPSS total score. Bland-Altman has the advantage of graphically depicting the agreement between test-retest measurements since the ICC provides statistical values. Adequate agreement is characterised by a mean difference that is close to zero and most points being within $95 \%$ limits of agreement of the difference in means.

Clinical utility of the Igbo-MSPSS was investigated using the standard error of measurement (SEM) and minimal detectable change (MDC). MDC is a statistical estimate that signifies a true change in a measured construct beyond measurement error. MDC was estimated using the standard error of measurement (SEM) and the measure's reliability as indicated in the equation below. SEM was obtained from the sample's standard deviation (SD) and the measure's test-retest reliability (R) using the equation below (de Vet et al., 2006).

$$
\begin{aligned}
& \mathrm{SEM}=\mathrm{SD} \sqrt{(1-\mathrm{R})} \\
& \mathrm{MDC}=1.96 \times \sqrt{2} \times \mathrm{SEM}
\end{aligned}
$$

(1.96 is from the $95 \%$ confidence interval, and $\sqrt{2}$ is from two measurements).

\section{Validity}

Validity assesses the degree to which an outcome tool measures what it is meant to measure (Tavakol \& Dennick, 2011). Construct validity evaluates how much a measure assesses its intended construct. As there is no 'gold standard' Igbo measure of social support, construct validity was assessed by investigating the predictive power of the adapted measure with constructs known to have established relationships with social support in the literature (criterion validity). The validity was assessed using Spearman's correlation analyses (the sample had a non-normal distribution) and were graded as not relevant $(<0.10)$, low $(0.10-0.30)$, moderate $(0.30-0.50)$, and high $(>0.50)$ (Cohen, 1988).

Igbo-MSPSS measures social support. It is therefore expected to have better correlation with getting along and cognition subscales of the Igbo-WHODAS whose constructs appear more closely associated with social support than the other Igbo-WHODAS subscales. The literature suggests that social support is not significantly correlated with pain intensity and pain-related disability in people with CLBP (Rzeszutek, Oniszczenko, Schier, Biernat-Kałuża, \& Gasik, 2016), therefore the IgboMSPSS is expected to have no significant correlation with Igbo-BS-11, Igbo-RMDQ, and the BPS.

Exploratory factor analysis (EFA) was used to determine the Igbo-MSPSS dimensionality (number of factors in the measure) with the Kaiser criterion and parallel analysis used to determine the number of factors to be retained. Kaiser Meyer Olkin (KMO) and Bartlett's test were employed using a minimum eigenvalue set at $\geq$ 1.00 for retention. Promax rotation, also known as oblique rotation, was done with the suppression of factor loadings less than 0.30 . This type of rotation supposes that factors in a measure can be related (Yong \& Pearce, 2013). Principal axis factoring was used to extract factors. The applied rotation method was Promax, and the factors were extracted using the maximum likelihood method. The inclusion of parallel analysis for determining the number of factors to be retained in the IgboMSPSS was to compensate for the weakness of the Kaiser criterion which can overestimate or underestimate the number of factors to be retained, in contrast to the parallel analyses which shows fewer fluctuations in its accuracy and is more robust (Coughlin, 2013; Sheytanova, 2015). Despite the fact that empirical guidelines are useful, they are not always correct. As the true number of factors is unknown in reality, it is suggested that several methods for estimating the number of factors be utilised and the meanings of findings explored (Coughlin, 2013; Sheytanova, 2015). The accuracy of empirical guidelines is more likely to be compromised when factors are highly correlated, factor loadings are low, the number of factors is large, and the sample size is small; hence, multiple criteria, including relevant theory and previous research, should be used to determine the number of factors to retain (Watkins, 2020). Watkins (2020) recommends selecting from among a set of competing theoretical explanations of the model that best balances the desirable characteristics of parsimony and fit to observed data in terms of interpretability and conceptual sense. Therefore, the number of factors of the adapted measure and their underlying associations were investigated and compared with the original MSPSS. Factor pattern coefficients were used for the factor loadings. Scree plots using the different methods for determining the number of factors to be extracted were additionally used for the visual exploration of the retained and excluded factors as recommended (Watkins, 2020).

\section{Floor and ceiling effects}

When a significant number of participants score the maximum or the least score on a measure, ceiling or floor effect occurs. This implies that the two extremes of 
the scale are not sufficiently differentiated. For this study, $15 \%$ or above was regarded as floor or ceiling effect (Lim et al., 2015).

\section{Results}

\section{Participant characteristics}

Table 1 describes the sociodemographic characteristics of all participant samples that participated in this study (cross-cultural adaptation, test-retest reliability, and validity samples). The sociodemographic characteristics of the test-retest and cross-sectional validity testing samples were similar except for a higher literacy level in the test-retest sample involving urban Nigerian populations. A wider applicability of the Igbo-MSPSS is assured by a more varied sample across different levels of literacy and habitation (rural and urban).

\section{Cross-cultural adaptation}

Translation, comprehensibility, and cultural equivalence of Igbo-MSPSS

Cross-cultural adaptation of the MSPSS was simple. The forward translation team decided on 'there is a special person that shares/partakes in my joys and my sorrows/ mourning' to reflect the original item 2 as 'sorrow' and 'mourning' are homographs in Igbo. A review by the expert committee found no ambiguous meanings throughout the translation. The Igbo-MSPSS was found easily understandable during field verbal pretesting with participants.

\section{Psychometric properties of Igbo-MSPSS}

There were no missing data in the psychometric analyses.

\section{Reliability}

Table 2 shows that the Cronbach's alpha and McDonald's $\omega$ were at least $>0.80$ for the Igbo-MSPSS and its subscales depicting moderate to high internal consistency. An ICC of 0.82 signifies very good intraclass correlation coefficient for the Igbo-MSPSS. SEM and MDC were 5.90 and 16.34 .

The test-retest values of the Igbo-MSPSS suggested adequate agreement with difference in mean near zero

Table 1 Demographic characteristics of all participants (cross-cultural adaptation, test-retest reliability, and criterion validity samples)

\begin{tabular}{|c|c|c|c|c|c|c|c|c|}
\hline & Age & Gender & $\begin{array}{l}\text { Marital } \\
\text { status }\end{array}$ & Main occupation & Religion & $\begin{array}{l}\begin{array}{l}\text { Education } \\
\text { (years } \\
\text { completed) }\end{array} \\
\end{array}$ & Literacy & Habitation \\
\hline $\begin{array}{l}\text { Cross-cultural adaptation } \\
\text { (pilot/pretesting) sample; } \boldsymbol{n} \\
=\mathbf{1 2}\end{array}$ & $\begin{array}{l}45 \text { years } \\
(S D 10.36)\end{array}$ & $\begin{array}{l}\text { Male: } 7 \\
(58.30 \%)\end{array}$ & $\begin{array}{l}\text { Married: } 11 \\
\text { (91.70\%) } \\
\text { Single: } 1 \\
(8.30 \%)\end{array}$ & $\begin{array}{l}\text { Non-manual workers: } \\
5(41.70 \%) \\
\text { Manual workers: } 7 \\
(58.30 \%)\end{array}$ & $\begin{array}{l}\text { Pentecostal: } \\
10 \text { (83.30\%) } \\
\text { Catholic: } 2 \\
(16.70 \%)\end{array}$ & $10.00(3.70)$ & $\begin{array}{l}\text { Illiterate: } 4 \\
\text { (33.30\%) } \\
\text { English: } 6 \\
\text { (50\%) } \\
\text { English/ } \\
\text { Igbo: } 2 \\
(16.70 \%)\end{array}$ & $\begin{array}{l}\text { Rural: } 12 \\
(100 \%)\end{array}$ \\
\hline $\begin{array}{l}\text { Test-retest reliability sample; } \\
\boldsymbol{n}=\mathbf{5 0}\end{array}$ & $\begin{array}{l}45.2 \text { years } \\
\text { (SD } 11.55)\end{array}$ & $\begin{array}{l}\text { Male: } 18 \\
(36.00 \%)\end{array}$ & $\begin{array}{l}\text { Married: } 37 \\
\text { (74.00\%) } \\
\text { Single: } 8 \\
\text { (16.00\%) } \\
\text { Widowed: } 4 \\
\text { (8.00\%) } \\
\text { Separated: } \\
1 \text { (2.00\%) }\end{array}$ & $\begin{array}{l}\text { Paid non-manual: } 25 \\
(50.00 \%) \\
\text { Self-employed } \\
\text { business/farming: } 19 \\
(38.00 \%) \\
\text { Keeping house/ } \\
\text { homemaker: } 2 \text { (4.00\%) } \\
\text { Student: } 2 \text { (4.00\%) } \\
\text { Non-paid work/ } \\
\text { volunteer/charity: } 1 \\
(2.00 \%)\end{array}$ & & $13.30(7.14)$ & & $\begin{array}{l}\text { Urban: } 30 \\
(60.00 \%) \\
\text { Rural: } 20 \\
(40.00 \%)\end{array}$ \\
\hline $\begin{array}{l}\text { Criterion validity sample; } \boldsymbol{n}= \\
200\end{array}$ & $\begin{array}{l}48.6 \text { years } \\
\text { (SD 12.00) }\end{array}$ & $\begin{array}{l}\text { Male: } \\
112 \\
(44.00 \%)\end{array}$ & $\begin{array}{l}\text { Married: } \\
143 \\
\text { (71.50\%) } \\
\text { Widowed: } \\
31(15.50 \%) \\
\text { Single: } 22 \\
\text { (11.00\%) } \\
\text { Cohabiting: } \\
2 \text { (1.00\%) } \\
\text { Separated: } \\
2 \text { (1.00\%) }\end{array}$ & $\begin{array}{l}\text { Self-employed } \\
\text { business/farming: } 125 \\
\text { (62.50\%) } \\
\text { Paid non-manual: } 31 \\
\text { (15.50\%) } \\
\text { Non-paid work/ } \\
\text { volunteer/charity: } 16 \\
(8.00 \%) \\
\text { Keeping house/ } \\
\text { homemaker: } 13 \\
\text { (6.50\%) } \\
\text { Student: } 7 \text { (3.50\%) } \\
\text { Unemployed (health } \\
\text { reasons): } 4 \text { (2.00\%) } \\
\text { Unemployed (other } \\
\text { reasons): } 3 \text { (1.50\%) } \\
\text { Retired: } 1 \text { (0.50\%) }\end{array}$ & & $7.00(6.40)$ & & $\begin{array}{l}\text { Rural: } 200 \\
(100 \%)\end{array}$ \\
\hline
\end{tabular}


Table 2 Reliability of Igbo-MSPSS

\begin{tabular}{|c|c|c|}
\hline & $\begin{array}{l}\text { Cronbach's alpha } \\
\text { All items: } 12 \text { (total score) }=0.88\end{array}$ & $\begin{array}{l}\text { McDonald's } \omega \\
\text { All items: } 12 \text { (total score) }=0.87\end{array}$ \\
\hline \multicolumn{3}{|l|}{ Factor structures } \\
\hline \multirow[t]{3}{*}{ Three-factor structure } & Factor 1 (friends) $=0.84$ & Factor 1 (friends) $=0.84$ \\
\hline & Factor 2 (significant other) $=0.87$ & Factor 2 (significant other) $=0.87$ \\
\hline & Factor 3 (family) $=0.89$ & Factor 3 (family) $=0.89$ \\
\hline \multirow[t]{2}{*}{ Two-factor structure } & Factor 1 (family and significant other) $=0.91$ & Factor 1 (family and significant other) $=0.91$ \\
\hline & Factor 2 (friends) $=0.84$ & Factor 2 (friends) $=0.84$ \\
\hline Item & Cronbach's alpha when item is removed & \\
\hline 1 & 0.87 & \\
\hline 2 & 0.87 & \\
\hline 3 & 0.87 & \\
\hline 4 & 0.87 & \\
\hline 5 & 0.86 & \\
\hline 6 & 0.87 & \\
\hline 7 & 0.88 & \\
\hline 8 & 0.88 & \\
\hline 9 & 0.87 & \\
\hline 10 & 0.86 & \\
\hline 11 & 0.86 & \\
\hline 12 & 0.88 & \\
\hline \multicolumn{3}{|l|}{ ICC (95\% Cl): $0.82(0.68,0.90)$} \\
\hline \multicolumn{3}{|l|}{ SEM: 5.90} \\
\hline MDC: 16.34 & & \\
\hline
\end{tabular}

and most points being within the $95 \%$ agreement limits of the difference in mean (Fig. 2).

\section{Criterion validity}

Igbo-MSPSS had a moderate negative correlation with getting along the subscale of the Igbo-WHODAS and a moderate positive correlation with the back performance scale. Igbo-MSPSS had a weak negative correlation with the Igbo-WHODAS (total score) and its subscales cognition, mobility, self-care, and participation and no significant correlation with pain intensity, self-reported back pain-specific disability, and life activities subscale of the Igbo-WHODAS (Table 3).

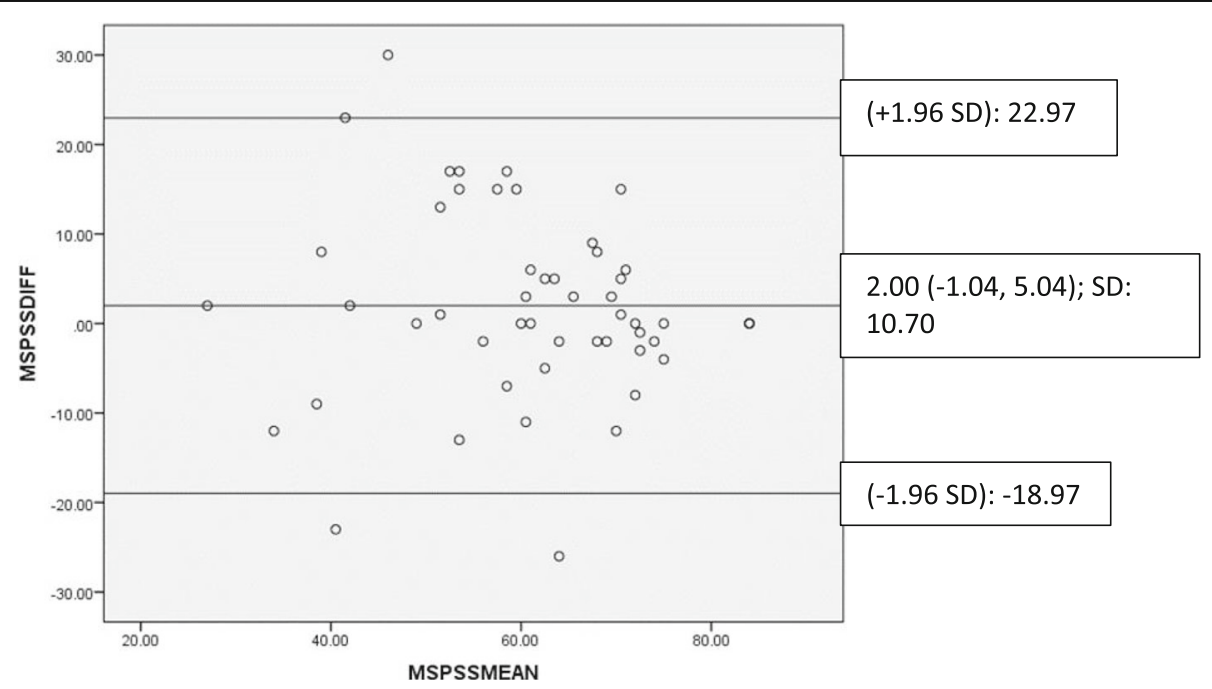

Fig. 2 Bland-Altman plot for test-retest agreement of the Igbo-MSPSS 


\section{Structural validity}

Table 4 shows a three-factor solution and a two-factor solution of the Igbo-MSPSS produced with the Kaiser criterion and parallel analysis respectively. The factor pattern did not change with the suppression of factor loadings less than 0.3. The same item-factor pattern emerged from an unconstrained EFA approach in the three-factor structure as in the original measure. The three-factor structure corresponded to the friends, significant other, and family subscales respectively in the original measure with no cross-loading of items. The two-factor structure corresponded to the original family and significant other subscales which loaded as one factor (factor 1), whilst factor 2 is the original friends' subscale with no cross-loading of items. The loadings (factor pattern coefficients) show larger values for 7 items in the three-factor structure, whereas larger loadings were observed for the remaining 5 items $(7,12,5$, 11, and 3) in the two-factor structure. Intercorrelations among the Igbo-MSPSS subscales for the three-factor structure depict high correlations between factor 2 (significant other) and factor 1 (friends), and between factor 3 (family) and factor 1 (friends), with a moderate correlation between factor 3 (family) and factor 2 (significant other). For the two-factor structure, there was a moderate correlation between factor 1 (family and significant other) and factor 2 (friends) (Table 4). Figures 3 and 4 also confirm a three-factor structure and a two-factor structure produced with the Kaiser criterion and the parallel analysis methods for determining dimensionality respectively.

\section{Findings from investigating floor and ceiling effects}

Zero $(0.00 \%)$ and $18(7.20 \%)$ participants scored 12 and 84 on the Igbo-MSPSS from the two samples (200

Table 3 Spearman's correlation analyses between Igbo-MSPSS and intensity of pain (BS-11), performance-based disability (BPS), self-reported back pain-specific disability (lgbo-RMDQ), selfreported generic disability (Igbo-WHODAS and its subscales)

\begin{tabular}{ll}
\hline & Igbo-MSPSS \\
\hline BS-11 (increasing pain intensity) & 0.00 \\
BPS (increasing performance-based disability) & $0.31^{* *}$ \\
Igbo-RMDQ (increasing back pain specific-disability) & 0.13 \\
lgbo-WHODAS (increasing generic disability) & $-0.23^{* *}$ \\
Difficulty with cognition & $-0.23^{* *}$ \\
Difficulty with mobility & $-0.15^{*}$ \\
Difficulty with self-care & $-0.17^{*}$ \\
Difficulty with getting along with people & $-0.32^{* *}$ \\
Difficulty with performing life activities & -0.09 \\
Difficulty with participation & $-0.14^{*}$ \\
\hline${ }^{*} p<0.05 ; * * 0.01$ &
\end{tabular}

${ }^{*} p<0.05 ;{ }^{* *} p<0.01$ participants for the criterion validity investigation, and 50 participants for the reliability assessments).

\section{Discussion}

Some English words in the original MSPSS had no exact Igbo equivalents; hence, Igbo phrases that retained the original conceptual meaning in the MSPSS were used in the Igbo-MSPSS. This could at least be partly explained in English being the official written language in Nigeria, whilst Igbo is mainly a spoken language in Nigeria. MSPSS was very easy to cross-culturally adapt and had good acceptability and comprehensibility supporting earlier studies (Akhtar et al., 2010; Chou, 2000; Ekbäck, Benzein, Lindberg, \& Årestedt, 2013; Wongpakaran, Wongpakaran, \& Ruktrakul, 2011). 'Sorrow' and 'mourning' are spelt similarly in Igbo, and the implication of both words in item 2 reflected the meaning in the original measure. The content validity of the IgboMSPSS was established by this cross-cultural adaptation.

The Igbo-MSPSS has a Cronbach's alpha of 0.88 corresponding exactly to the original MSPSS (Zimet et al., 1988, 1990), similar in Swedish, Chinese, Pakistani, Thai, Urdu, and Nepali cultures (Akhtar et al., 2010; Chou, 2000; Ekbäck et al., 2013; Wongpakaran et al., 2011). The test-retest reliability of the Igbo-MSPSS appeared sound with ICC scores of $0.82(0.68,0.90)$ and BlandAltman plots that showed good agreement. This concurs with the original MSPSS and other translations (Akhtar et al., 2010; Chou, 2000; Ekbäck et al., 2013; Tonsing, Zimet, \& Tse, 2012; Wongpakaran et al., 2011). There is no established minimal clinically important difference (MCID) with which to compare the SEM and MDC of the Igbo-MSPSS, although this may likely be contextspecific.

Aspects of the criterion validity of the Igbo-MSPSS was supported to a small extent. There was a lack of correlation of Igbo-MSPSS with pain intensity, self-reported back pain-specific disability and life activities subscale of the Igbo-WHODAS (generic self-reported disability). Evidence suggests that social support may not be directly associated with pain intensity and self-reported back pain-specific disability in people with CLBP (Rzeszutek et al., 2016; Suner, 2007), but may be directly associated with psychological outcomes such as depression and unhelpful coping strategies, such as kinesiophobia, and catastrophising in people with CLBP (Campbelll, WynneJones, \& Dunn, 2011; López-Martínez, Esteve-Zarazaga, \& Ramírez-Maestre, 2008; McKillop, Carroll, Jones, \& Battié, 2017). Furthermore, the life activities subscale of the WHODAS (Igwesi-Chidobe et al., 2020) measures the ease or difficulty with which common household tasks and work/school activities are performed, which are the concepts mainly captured in self-reported back pain-specific disability measured with the RMDQ 
Table 4 Factor loading of Igbo-MSPSS items from exploratory factor analysis using the Kaiser criterion and parallel analysis for determining dimensionality compared with original MSPSS and intercorrelations among the subscales of the lgbo-MSPSS for the three-factor and two factor structures

\begin{tabular}{|c|c|c|c|}
\hline & Factor 1 (friends) & Factor 2 (significant other) & Factor 3 (family) \\
\hline \multicolumn{4}{|l|}{ Factor structures of the Igbo-MSPSS } \\
\hline \multicolumn{4}{|l|}{ Igbo-MSPSS } \\
\hline Igbo-MSPSS6 & $\mathrm{KC} / \mathrm{PA}=.84 / .65(\mathrm{MSPSS}=.82)$ & & \\
\hline Igbo-MSPSS9 & $\mathrm{KC} / \mathrm{PA}=.79 / .71(\mathrm{MSPSS}=.86)$ & & \\
\hline Igbo-MSPSS7 & $\mathrm{KC} / \mathrm{PA}=.77 / .90(\mathrm{MSPSS}=.79)$ & & \\
\hline Igbo-MSPSS12 & $\mathrm{KC} / \mathrm{PA}=.74 / .78(\mathrm{MSPSS}=.86)$ & & \\
\hline Igbo-MSPSS2 & & $\mathrm{KC} / \mathrm{PA}=.92 / .71(\mathrm{MSPSS}=.91)$ & \\
\hline Igbo-MSPSS1 & & $\mathrm{KC} / \mathrm{PA}=.90 / .77(\mathrm{MSPSS}=.74)$ & \\
\hline Igbo-MSPSS10 & & $\mathrm{KC} / \mathrm{PA}=.75 / .69(\mathrm{MSPSS}=.92)$ & \\
\hline Igbo-MSPSS5 & & $\mathrm{KC} / \mathrm{PA}=.62 / .74(\mathrm{MSPSS}=.91)$ & \\
\hline Igbo-MSPSS8 & & & $\mathrm{KC} / \mathrm{PA}=.83 / .70(\mathrm{MSPSS}=.84)$ \\
\hline Igbo-MSPSS4 & & & $\mathrm{KC} / \mathrm{PA}=.78 / .69(\mathrm{MSPSS}=.84)$ \\
\hline Igbo-MSPSS11 & & & $\mathrm{KC} / \mathrm{PA}=.74 / .87(\mathrm{MSPSS}=.81)$ \\
\hline Igbo-MSPSS3 & & & $\mathrm{KC} / \mathrm{PA}=.70 / .87(\mathrm{MSPSS}=.83)$ \\
\hline \multicolumn{4}{|l|}{$\mathrm{KMO}=0.88$ (Kaiser criterion) } \\
\hline \multicolumn{4}{|l|}{$X^{2}=1415.25^{* * *}$ (Kaiser criterion) } \\
\hline \multicolumn{4}{|c|}{ Intercorrelations among Igbo-MSPSS subscales } \\
\hline Three-factor structure & Factor 1 (friends) & Factor 2 (significant other) & Factor 3 (family) \\
\hline Factor 1 (friends) & 1.00 & 0.64 & 0.63 \\
\hline Factor 2 (significant other) & & 1.00 & 0.37 \\
\hline Factor 3 (family) & & & 1.00 \\
\hline Two-factor structure & Factor 1 (family and significant other) & Factor 2 (friends) & \\
\hline Factor 1 (family and significant other) & 1.00 & 0.38 & \\
\hline Factor 2 (friends) & & 1.00 & \\
\hline
\end{tabular}

KC Kaiser criterion and PA parallel analysis: both used for determining number of factors to be extracted. ${ }^{* * *} p<0.001 ;$ Factor loadings $\geq 0.30$ are presented; KMO Kaiser-Meyer-Olkin and is a measure of adequacy of sampling; $x^{2}=$ Bartlett's sphericity test using chi-square; Principal axis factoring extraction; Promax with Kaiser normalization rotation; 5 iterations for rotation convergence; MSPSS = factor loadings from the original MSPSS study

(Igwesi-Chidobe et al., 2019; Roland \& Morris, 1983). However, correlations with getting along and cognition subscales of the Igbo-WHODAS which are not direct social support constructs is an important limitation of this study. As other Igbo measures of social support are developed, they can be used to confirm the construct validity of the Igbo-MSPSS via convergent validity investigations in future studies.

The moderate negative correlation with getting along subscale of the Igbo-WHODAS shows that the higher the social support measured by Igbo-MSPSS, the lower the difficulties encountered in getting along with people, a subscale of the Igbo-WHODAS which closely reflects the social support construct as it measures the ease or difficulty with which people interact with others socially.

The weak negative correlation of the Igbo-MSPSS with the Igbo-WHODAS (total score) and its subscales of cognition, mobility, self-care, and participation could be because these contained aspects of social support and/or psychological constructs known to be associated with social support. For example, the cognition subscale of the Igbo-WHODAS explores the ability to concentrate, remember, analyse, learn, understand, and start/maintain a conversation which are psychological constructs, a higher score of which signifies more difficulty with the activity. This explains the negative correlation with IgboMSPSS, a higher score of which signifies more social support. Starting/maintaining a conversation may also be reflective of social support as people with limited social circle may have limited opportunities to have conversations. The mobility and self-care subscales of the Igbo-WHODAS may have reflected both emotional state and social support in people living with CLBP in this population with CLBP that tend to remain indoors (and therefore unlikely to dress up) worrying about their problems when they are psychologically distressed or feel unsupported by family and friends (Igwesi-Chidobe, Kitchen, et al., 2017). The participation subscale of the 


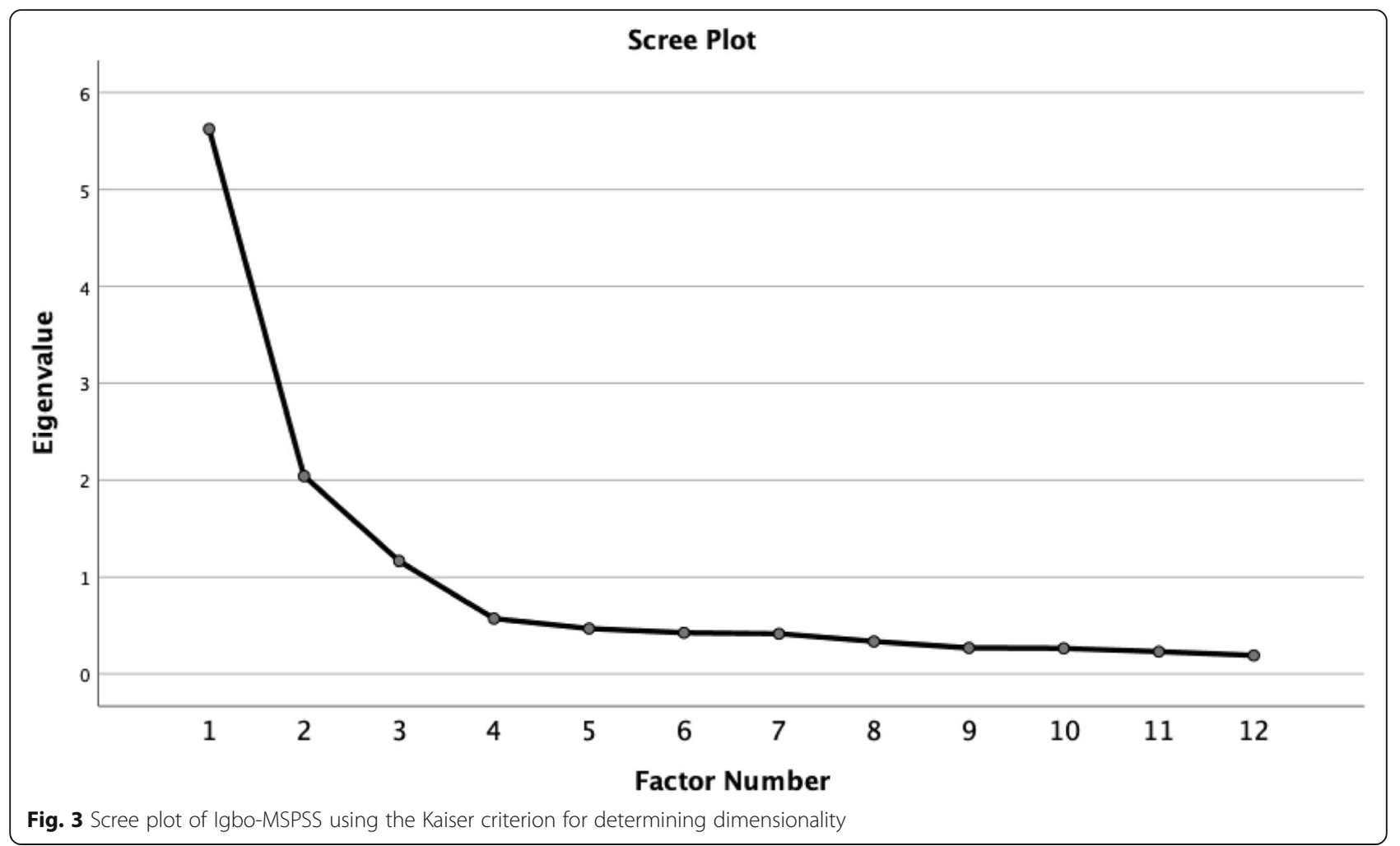

Igbo-WHODAS evaluates the extent to which the participants are able to join in community activities, live with dignity in their community, are emotionally affected by their back pain, have financial and family problems and have difficulties relaxing or having pleasure. These are concepts represented in social support and emotional distress explaining the association with Igbo-MSPSS.

The moderate positive correlation of the Igbo-MSPSS with the back performance scale could mean that increased social support resulted from the limitation in

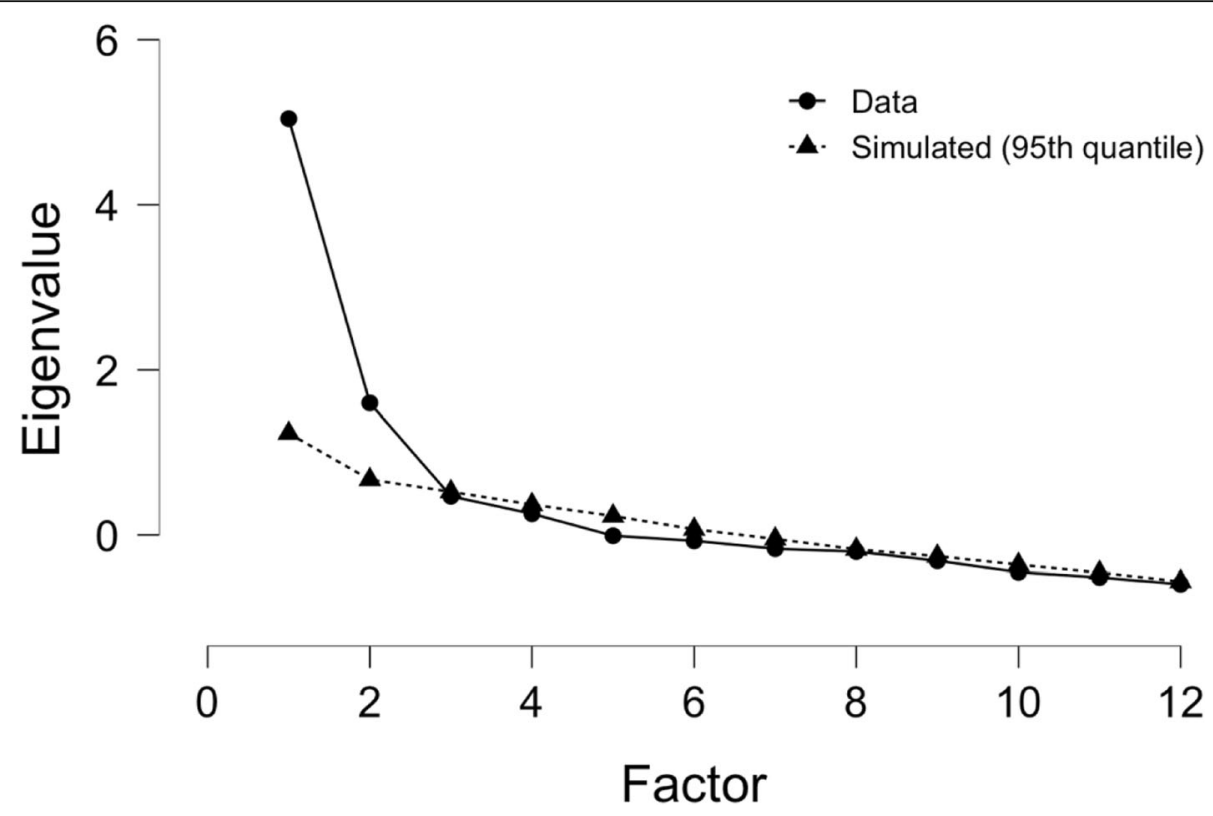

Fig. 4 Scree plot of Igbo-MSPSS using the parallel analysis for determining dimensionality 
the mobility of people in this population as social support is a method of coping assistance (Igwesi-Chidobe, Coker, et al., 2017). Social support has also been shown to be a source of stress as social relationships are inherently double-edged with blatantly unsupportive behaviour often reported from others (Taylor, 2011).

The structural validity of the Igbo-MSPSS was reinforced by the reproduction of the same item-factor pattern in the three-factor structure which emerged from an unconstrained EFA as in the original MSPSS (Zimet et al., 1988). This is similar to other adaptations (Ekbäck et al., 2013; Tonsing et al., 2012; Wongpakaran et al., 2011) but contradicts the one-factor structure observed in Pakistan (Akhtar et al., 2010) and the two-factor structure in Hong Kong (Chou, 2000). This likely represents cultural differences in the interpretation of social support in these populations. Another reason could be the statistical methods applied. Performing the exploratory factor analysis of the Igbo-MSPSS using the parallel analysis rather than the Kaiser method (which often overestimates the number of factors to be retained) to determine dimensionality, produced a two-factor structure. Family and significant other subscales were represented as one factor, whilst the friends subscale was the other factor. This suggests that support from family and significant other may not be distinct in this population or that significant others may also be family members in this population. This aligns with findings in the Arabic and Turkish populations where significant other was interpreted as spouse or husband (Dambi et al., 2018). This outcome does not support the findings from the three-factor structure of the Igbo-HADS where a much lower correlation $(r=0.37)$ was observed between factor 3 (family) and factor 2 (significant other), than between factor 2 (significant other) and factor 1 (friends) $(r=$ 0.64 ), and between factor 3 (family) and factor 1 (friends) $(r=0.63)$. The level of correlation between factor 2 (significant other) and factor 1 (friends) $(r=0.64)$ was similar to the correlation between factor 3 (family) and factor 1 (friends) $(r=0.63)$ in the three-factor structure. Additionally, the level of correlation between factor 1 (family and significant other) and factor 2 (friends) $(r$ $=0.38)$ in the two-factor structure was similar to the correlation between family and significant other $(r=$ 0.37 ) in the three-factor structure. The above results suggest that significant others can be family in some instances and friends in some other situations, and that members of a family can be sometimes be regarded as friends. This may be related to the African culture where distant members of a family tree can still be regarded as family. The factor pattern coefficient loadings were greater for 7 items which included three significant other items (1: special person around when in need, 2: special person with whom to share joys and sorrow, 10: special person that cares about participants' feelings), two family items (4: getting emotional help and support from family, 8: talking about problems with family), and two friends items (6: friends trying to help, 9: having friends with whom to share joys and sorrows) in the threefactor structure. In the two-factor structure, greater factor pattern coefficient loadings were observed for the remaining 5 items which included two friends items (7: being able to count on friends when things go wrong, and 12: being able to talk about problems with friends), two family items (having family that is willing to help with decision-making, and having family that tries to help) and one significant other item (having a special person who is a source of comfort). Overall, these findings suggest that the three-factor structure of the IgboMSPSS may have better applicability in this population than the two-factor structure. The MSPSS can yield one (as suggested in the literature), two or three factors (as shown in this study and the literature). Therefore, it is recommended that the utility of these possible structures is confirmed in this population using confirmatory factor analysis of the Igbo-MSPSS in future studies utilising much larger sample sizes.

The development of a valid and reliable measure of social support for Igbo speaking populations, particularly those that are illiterate, have been made possible by this study. Studies investigating the sensitivity-to-change of the IgboMSPSS would be required to determine its MCID using analysis such as receiver operating characteristic (ROC) curves. Such analysis includes patients' individual global impression of change (Stratford \& Riddle, 2005).

A limitation of this study was the lack of bilingual assessment of item-by-item agreement between the original MSPSS and Igbo-MSPSS. This should be conducted by future studies involving in populations with sufficient literacy to enable understanding of English and Igbo. Additionally, confirmatory factor analysis of the Igbo-MSPSS is warranted to confirm the factor structure of the Igbo-MSPSS using much larger sample sizes in different populations in Nigeria, particularly in the case that only a few items of the Igbo-MSPSS have high factor loading scores (>0.80) (Yong \& Pearce, 2013). However, correlations of the Igbo-MSPSS with constructs that align with social support in the literature support its construct validity to some extent. The correlation coefficients in this study must be interpreted as exploratory rather than confirmatory until future studies utilising much larger sample sizes confirm the reported correlations between Igbo-MSPSS and the stated outcomes.

\section{Conclusions}

The Igbo-MSPSS appears to be a valid and reliable measure of social support in Nigeria. Further research is 
required to confirm its validity, reliability, and utility in Nigeria.

\section{Supplementary Information}

The online version contains supplementary material available at https://doi. org/10.1186/s42409-021-00026-7.

Additional file 1: Appendix 1. The Igbo Multidimensional Scale of Perceived Social Support (Igbo-MSPSS). Appendix 2. Original Multidimensional Scale of Perceived Social Support (MSPSS).

\section{Acknowledgments}

The authors are grateful to the participants for providing data for this study.

\section{Authors' contributions}

CNI-C conceived of this work, designed the study, collected data, conducted analyses, interpreted findings, and drafted the initial manuscript. RCM contributed to the collection of data and interpretation of findings. ELG and IOS contributed to the interpretation of findings. EG supervised data collection. The authors read, provided feedback, and approved the final version of the manuscript to be published.

\section{Funding}

This study was funded by the Tertiary Education Trust Fund, Nigeria and the Schlumberger Foundation, The Netherlands. Both organizations had no influence on the study design; in the collection, analysis and interpretation of data; in the writing of the report; and in the decision to submit the manuscript for publication.

\section{Availability of data and materials}

Data are available on request due to ethical restrictions imposed by Biomedical \& Health Sciences, Dentistry, Medicine and Natural \& Mathematical Sciences Research Ethics Subcommittees (BDM RESC) King's College London. Requests for data access may be made to BDM RESC King's College London through email bdm@kcl.ac.uk.

\section{Declarations}

\section{Ethics approval and consent to participate}

King's College London (Ref: BDM/13/14-99) and University of Nigeria Teaching Hospital (Ref: UNTH/CSA/329/Nol.5) gave ethical approvals. Verbal and written consents were obtained from all participants. Interested participants signed or thumb printed on the consent forms following a detailed verbal and written explanation of the study and after being given three days to decide whether to participate in the study.

\section{Consent for publication}

Not applicable.

\section{Competing interests}

The authors declare that they have no competing interests.

\section{Author details}

${ }^{1}$ Department of Medical Rehabilitation, Faculty of Health Sciences and Technology, College of Medicine, University of Nigeria (Enugu Campus), Enugu, Nigeria. ${ }^{2}$ Department of Physiotherapy, School of Population Health Sciences, Faculty of Life Sciences and Medicine, King's College London, London, UK. ${ }^{3}$ Department of Psychology, Institute of Psychiatry, Psychology and Neuroscience, King's College London, London, UK.

\section{Received: 2 November 2020 Accepted: 1 June 2021}

\section{Published online: 25 June 2021}

\section{References}

Adamczyk, K. (2013). Development and validation of the Polish-language version of the Multidimensional Scale of Perceived Social Support (MSPSS). Revue Internationale de Psychologie Sociale, 26(4), 25-48.

Akhtar, A., Rahman, A., Husain, M., Chaudhry, I. B., Duddu, V., \& Husain, N. (2010). Multidimensional scale of perceived social support: Psychometric properties in a South Asian population. Journal of Obstetrics and Gynaecology Research, 36(4), 845-851. https://doi.org/10.1111/j.1447-0756.2010.01204.X.

Beaton, D. E., Bombardier, C., Guillemin, F., \& Ferraz, M. B. (2000). Guidelines for the process of cross-cultural adaptation of self-report measures. Spine, 25(24), 3186-3191. https://doi.org/10.1097/00007632-200012150-00014.

Bland, J. M., \& Altman, D. (1986). Statistical methods for assessing agreement between two methods of clinical measurement. The Lancet, 327(8476), 307310. https://doi.org/10.1016/S0140-6736(86)90837-8.

Bujang, M. A., \& Baharum, N. (2017). A simplified guide to determination of sample size requirements for estimating the value of intraclass correlation coefficient: A review. Archives of Orofacial Science, 12(1), 1-11.

Campbell, P., Wynne-Jones, G., Muller, S., \& Dunn, K. M. (2013). The influence of employment social support for risk and prognosis in nonspecific back pain: A systematic review and critical synthesis. International Archives of Occupational and Environmental Health, 86(2), 119-137. https://doi.org/10.1007/s00420-0120804-2.

Campbelll, P., Wynne-Jones, G., \& Dunn, K. M. (2011). The influence of informal social support on risk and prognosis in spinal pain: A systematic review. European Journal of Pain, 15(5), 444-4e1.

Chou, K.-L. (2000). Assessing Chinese adolescents' social support: The multidimensional scale of perceived social support. Personality and Individual Differences, 28(2), 299-307. https://doi.org/10.1016/S0191-8869(99)00098-7.

Cohen, J. (1988). Set correlation and contingency tables. Applied Psychological Measurement, 12(4), 425-434. https://doi.org/10.1177/014662168801200410.

Coughlin, K. B. (2013). An analysis of factor extraction strategies: A comparison of the relative strengths of principal axis, ordinary least squares, and maximum likelihood in research contexts that include both categorical and continuous variables.

Dambi, J. M., Corten, L., Chiwaridzo, M., Jack, H., Mlambo, T., \& Jelsma, J. (2018). A systematic review of the psychometric properties of the cross-cultural translations and adaptations of the Multidimensional Perceived Social Support Scale (MSPSS). Health and Quality of Life Outcomes, 16(1), 1-19.

De Maria, M., Vellone, E., Durante, A., Biagioli, V., \& Matarese, M. (2018). Psychometrics evaluation of the Multidimensional Scale of Perceived Social Support (MSPSS) in people with chronic disease. Annali Dell'Istituto Superiore Di Sanita, 54(4), 308-315. https://doi.org/10.4415/ANN_18_04 07.

de Vet, H. C., Terwee, C. B., Ostelo, R. W., Beckerman, H., Knol, D. L., \& Bouter, L. M. (2006). Minimal changes in health status questionnaires: Distinction between minimally detectable change and minimally important change. Health and Quality of Life Outcomes, 4(1), 54. https://doi.org/10.1186/1477-7525-4-54.

Ehrlich George, E. (2003). Low back pain/Ehrlich George E. Bulletin of the World Health Organization, 81, 671-676.

Ekbäck, M., Benzein, E., Lindberg, M., \& Årestedt, K. (2013). The Swedish version of the multidimensional scale of perceived social support (MSPSS)-a psychometric evaluation study in women with hirsutism and nursing students. Health and Quality of Life Outcomes, 11(1), 168. https://doi.org/10.11 86/1477-7525-11-168

Gabardo-Martins, L. M. D., Ferreira, M. C., \& Valentini, F. (2017). Psychometric properties of the multidimensional scale of perceived social support. Trends in Psychology, 25(4), 1873-1883.

Grotle, M., Brox, J. I., \& Vollestad, N. K. (2003). Cross-cultural adaptation of the Norwegian versions of the Roland-Morris Disability Questionnaire and the Oswestry Disability Index. Journal of Rehabilitation Medicine, 35(5), 241-247. https://doi.org/10.1080/16501970306094.

Hawker, G. A. Mian, S. Kendzerska, T. \& French, M. (2011). Measures of adult pain: Visual analog scale for pain (VAS pain), numeric rating scale for pain (NRS pain), McGill pain questionnaire (MPQ), short-form McGill pain questionnaire (SF-MPQ), chronic pain grade scale (CPGS), short form-36 bodily pain scale (SF-36 BPS). Arthritis Care \& Research, 63(S11), S240-S252.

Igwesi-Chidobe, C. N., Coker, B., Onwasigwe, C. N., Sorinola, I. O., \& Godfrey, E. L. (2017). Biopsychosocial factors associated with chronic low back pain disability in rural Nigeria: A population-based cross-sectional study. BMJ Global Health, 2(3), e000284. https://doi.org/10.1136/bmjgh-2017-000284.

Igwesi-Chidobe, C. N., Kitchen, S., Sorinola, I. O., \& Godfrey, E. L. (2017). "A life of living death": The experiences of people living with chronic low back pain in rural Nigeria. Disability and Rehabilitation, 39(8), 779-790. https://doi.org/10.31 09/09638288.2016.1161844.

Igwesi-Chidobe, C. N., Kitchen, S., Sorinola, I. O., \& Godfrey, E. L. (2020). World Health Organisation Disability Assessment Schedule (WHODAS 2.0): Development and validation of the Nigerian Igbo version in patients with chronic low back pain. BMC Musculoskeletal Disorders, 21(1), 1-14. 
Igwesi-Chidobe, C. N., Obiekwe, C., Sorinola, I. O., \& Godfrey, E. L. (2019). Assessing self-reported disability in a low-literate population with chronic low back pain: Cross-cultural adaptation and psychometric testing of Igbo Roland Morris disability questionnaire. Disability and Rehabilitation, 41(8), 948-957. https://doi.org/10.1080/09638288.2017.1416185.

Igwesi-Chidobe, C. N., Sorinola, I. O., Kitchen, S., \& Godfrey, E. L. (2018). Unconventional practitioners' causal beliefs and treatment strategies for chronic low back pain in rural Nigeria. Health Services Insights, 11, 117863291880878. https://doi.org/10.1177/1178632918808783.

Jensen, M. P., Karoly, P., \& Braver, S. (1986). The measurement of clinical pain intensity: A comparison of six methods. Pain, 27(1), 117-126. https://doi.org/1 0.1016/0304-3959(86)90228-9.

Koo, T. K., \& Li, M. Y. (2016). A guideline of selecting and reporting intraclass correlation coefficients for reliability research. Journal of Chiropractic Medicine, 15(2), 155-163. https://doi.org/10.1016/j.jcm.2016.02.012.

Laksmita, O. D., Chung, M.-H., Liao, Y.-M., \& Chang, P.-C. (2020). Multidimensional Scale of Perceived Social Support in Indonesian adolescent disaster survivors: A psychometric evaluation. PLoS One, 15(3), e0229958. https://doi.org/10.13 71/journal.pone.0229958.

Lee, S. C., Moy, F. M., \& Hairi, N. N. (2017). Validity and reliability of the Malay version multidimensional scale of perceived social support (MSPSS-M) among teachers. Quality of Life Research, 26(1), 221-227. https://doi.org/10.1 007/s11136-016-1348-9.

Lim, C. R., Harris, K., Dawson, J., Beard, D. J., Fitzpatrick, R., \& Price, A. J. (2015). Floor and ceiling effects in the OHS: An analysis of the NHS PROMs data set. BMJ Open, 5(7), e007765. https://doi.org/10.1136/bmjopen-2015-007765.

Lopez Ramos, Y., Fernandez Munoz, J. J., Navarro-Pardo, E., \& Murphy, M. (2017). Confirmatory factor analysis for the multidimensional scale of perceived social support in a sample of early retirees enrolled in university programs. Clinical Gerontologist, 40(4), 241-248. https://doi.org/10.1080/07317115.201 6.1199077.

López-Martínez, A. E., Esteve-Zarazaga, R., \& Ramírez-Maestre, C. (2008). Perceived social support and coping responses are independent variables explaining pain adjustment among chronic pain patients. The Journal of Pain, 9(4), 373379. https://doi.org/10.1016/j.jpain.2007.12.002.

Magnussen, L., Strand, L. I., \& Lygren, H. (2004). Reliability and validity of the back performance scale: Observing activity limitation in patients with back pain. Spine, 29(8), 903-907. https://doi.org/10.1097/00007632-200404150-00017.

McKillop, A. B., Carroll, L. J., Jones, C. A., \& Battié, M. C. (2017). The relation of social support and depression in patients with chronic low back pain. Disability and Rehabilitation, 39(15), 1482-1488. https://doi.org/10.1080/0963 8288.2016.1202335.

Mokkink, L. B., Prinsen, C. A. C., Patrick, D. L., Alonso, J., Bouter, L. M., De Vet, H. C., \& Terwee, C. B. (2019). COSMIN study design checklist for Patient-reported outcome measurement instruments. Department of Epidemiology and Biostatistics.

Mundfrom, D. J., Shaw, D. G., \& Ke, T. L. (2005). Minimum sample size recommendations for conducting factor analyses. International Journal of Testing, 5(2), 159-168. https://doi.org/10.1207/s15327574ijt0502_4.

Myklebust, M., Magnussen, L., \& Inger Strand, L. (2007). Back Performance Scale scores in people without back pain: Normative data. Advances in Physiotherapy, 9(1), 2-9. https://doi.org/10.1080/14038190601090794.

Pearson, J. E. (1986). The definition and measurement of social support. Journal of Counseling \& Development, 64(6), 390-395. https://doi.org/10.1002/j.15566676.1986.tb01144.x.

Roland, M., \& Fairbank, J. (2000). The Roland-Morris disability questionnaire and the Oswestry disability questionnaire. Spine, 25(24), 3115-3124. https://doi. org/10.1097/00007632-200012150-00006.

Roland, M., \& Morris, R. (1983). A study of the natural history of back pain: Part 1: Development of a reliable and sensitive measure of disability in low-back pain. Spine, 8(2), 145-150. https://doi.org/10.1097/00007632-19830300000005.

Rzeszutek, M., Oniszczenko, W., Schier, K., Biernat-Kałuża, E., \& Gasik, R. (2016). Pain intensity, temperament traits and social support as determinants of trauma symptoms in patients suffering from rheumatoid arthritis and low-back pain. International Journal of Rheumatic Diseases, 19(4), 412-419. https://doi.org/1 0.1111/1756-185X.12784.

Schönbrodt, F. D., \& Perugini, M. (2013). At what sample size do correlations stabilize? Journal of Research in Personality, 47(5), 609-612. https://doi.org/10.1 016/j.jp.2013.05.009.
Schönbrodt, F. D., \& Perugini, M. (2018). "At what sample size do correlations stabilize?": Corrigendum.

Sheytanova, T. (2015). A Monte Carlo study comparing three methods for determining the number of principal components and factors.

Shrout, P. E., \& Fleiss, J. L. (1979). Intraclass correlations: Uses in assessing rater reliability. Psychological Bulletin, 86(2), 420-428. https://doi.org/10.1037/0033-2 909.86.2.420

Strand, L. I., Moe-Nilssen, R., \& Ljunggren, A. E. (2002). Back Performance Scale for the assessment of mobility-related activities in people with back pain. Physical Therapy, 82(12), 1213-1223. https://doi.org/10.1093/ptj/82.12.1213.

Stratford, P. W., \& Riddle, D. L. (2005). Assessing sensitivity to change: Choosing the appropriate change coefficient. Health and Quality of Life Outcomes, 3(1), $1-7$.

Suner, N. A. A. (2007). The relationship between perceived family support, pain comprehension, catastrophizing and pain intensity among chronic pain patients in Selayang Hospital. Kulliyyah of Islamic Revealed Knowledge and Human Sciences, International.

Tavakol, M., \& Dennick, R. (2011). Making sense of Cronbach's alpha. International Journal of Medical Education, 2, 53-55. https://doi.org/10.5116/ijme.4dfb.8dfd.

Taylor, S. E. (2011). Social support: A review.

Thoits, P. A. (2011). Mechanisms linking social ties and support to physical and mental health. Journal of Health and Social Behavior, 52(2), 145-161. https:// doi.org/10.1177/0022146510395592.

Tonsing, K., Zimet, G. D., \& Tse, S. (2012). Assessing social support among South Asians: The multidimensional scale of perceived social support. Asian Journal of Psychiatry, 5(2), 164-168. https://doi.org/10.1016/j.ajp.2012.02.012.

Trejos-Herrera, A. M., Bahamón, M. J., Alarcón-Vásquez, Y., Vélez, J. I., \& Vinaccia, S. (2018). Validity and reliability of the multidimensional scale of perceived social support in Colombian adolescents. Psychosocial Intervention, 27(1), 5663. https://doi.org/10.5093/pi2018a1.

Üstün, T. B., Kostanjsek, N., Chatterji, S., \& Rehm, J. (2010). Measuring health and disability: Manual for WHO disability assessment schedule WHODAS 2.0. World Health Organization.

Villumsen, M., Holtermann, A., Samani, A., \& Madeleine, P. (2016). Social support modifies association between forward bending of the trunk and low-back pain: Cross-sectional field study of blue-collar workers. Scandinavian Journal of Work, Environment \& Health, 42(2), 125-134. https://doi.org/10.5271/sjweh.3 549

Watkins, M. W. (2020). A step-by-step guide to exploratory factor analysis with $R$ and RStudio. Routledge. https://doi.org/10.4324/9781003120001.

Wild, D., Grove, A., Martin, M., Eremenco, S., McElroy, S., Verjee-Lorenz, A., \& Erikson, P. (2005). Principles of good practice for the translation and cultural adaptation process for patient-reported outcomes (PRO) measures: Report of the ISPOR task force for translation and cultural adaptation. Value in Health, 8(2), 94-104. https://doi.org/10.1111/j.1524-4733.2005.04054.x.

Wongpakaran, T., Wongpakaran, N., \& Ruktrakul, R. (2011). Reliability and validity of the multidimensional scale of perceived social support (MSPSS): Thai version. Clinical Practice and Epidemiology in Mental Health: CP \& EMH, 7(1), 161-166. https://doi.org/10.2174/1745017901107010161.

World Health Organisation (2001). International classification of functioning, disability and health: ICF. World Health Organisation.

Yong, A. G., \& Pearce, S. (2013). A beginner's guide to factor analysis: Focusing on exploratory factor analysis. Tutorials in Quantitative Methods for Psychology, 9(2), 79-94. https://doi.org/10.20982/tqmp.09.2.p079.

Zimet, G. D., Dahlem, N. W., Zimet, S. G., \& Farley, G. K. (1988). The multidimensional scale of perceived social support. Journal of Personality Assessment, 52(1), 30-41. https://doi.org/10.1207/s15327752jpa5201_2.

Zimet, G. D., Powell, S. S., Farley, G. K., Werkman, S., \& Berkoff, K. A. (1990). Psychometric characteristics of the multidimensional scale of perceived social support. Journal of Personality Assessment, 55(3-4), 610-617. https://doi.org/1 0.1207/s15327752jpa5503\&4_17.

\section{Publisher's Note}

Springer Nature remains neutral with regard to jurisdictional claims in published maps and institutional affiliations. 